\title{
A detailed spatiokinematic model of the multipolar planetary nebula NGC 7026
}

\author{
David M. Clark, Jose Alberto López, Wolfgang Steffen and \\ Michael G. Richer \\ Instituto de Astronomía, Universidad Nacional Autónoma de México, Campus Ensenada, \\ Ensenada, Baja California, 22800, Mexico \\ e-mail: dmclark@astrosen.unam.mx, jal@astrosen.unam.mx, wsteffen@astrosen.unam.mx, \\ richer@astrosen.unam.mx
}

\begin{abstract}
We present the most extensive, long-slit, high-resolution coverage of the complex planetary nebula (PN), NGC 7026. Ten spectra were acquired using the Manchester Echelle Spectrometer at San Pedro Martir Observatory in Baja California, Mexico, and each shows exquisite detail, revealing the intricate structure of this object. Incorporating these spectra into the 3-dimensional visualization and kinematic program, Shape, and using HST images of NGC 7026, we have produced a detailed structural and kinematic model of this PN. Knowledge of the 3 -D structure of this nebula is relevant to understand the physics behind the extended X-ray emission in this object.
\end{abstract}

Keywords. ISM: kinematics and dynamics, planetary nebulae: general, planetary nebulae: individual (NGC 7026)

\section{Introduction}

NGC 7026 is a multi-polar planetary nebula $(\mathrm{PN})$ with a complex emission structure. Past observations indicate this PN consists of two sets of bipolar lobes (Cuesta et al. 1996). These authors suggest it is a bipolar nebula at an early, evolutionary stage. NGC 7026 was also observed in X-rays (Gruendl et al. 2006). It is one of the brightest, X-rayemitting PN known with a plasma temperature of $T=1.1 \times 10^{6} \mathrm{~K}$.

\section{Morphology, Kinematics and 3-D Structure}

Both ground and spaced-based observations show NGC 7026 to have an intricate structure. In [N II], HST images, the high spatial resolution of this telescope reveals a filamentary structure of the lobes and shows diffuse emission outside of the equatorial region (Figure 1, right, a)). In the mid-infrared, Spitzer $8 \mu \mathrm{m}$ observations show the central region has a considerable amount of dust (Figure 1, right, b)). But Solf \& Weinberger (1984) mention that this PN is optically thin. A ground-based, $60 \mathrm{~s} \mathrm{H} \alpha$ image acquired with MES-SPM shows NGC 7026 contains a bright equatorial region with complex lobes extending to the $\mathrm{N}$ and $\mathrm{S}$ of the nucleus (Figure 1, right, c)).

The [N II] $\lambda 6583 \AA$ line shows the most detail and was used in our 3-D reconstruction of the PN. Using slit $\mathrm{f}$ (Figure 1, left), we found a heliocentric, systemic velocity of $41.5 \mathrm{~km} \mathrm{~s}^{-1}$ and all quoted velocities henceforth are with respect to this velocity. We used the program Shape (Steffen \& López 2006, Steffen et al. 2011) to make our 3-D reconstruction (Figure 1, right, d)).

We found that NGC 7026 can best be described using three lobes to the S and three lobes to the N. The largest lobes are to the SE and NW. Smaller lobes, one in front and one in back, are seen to the SW and NE. The lobes are not distinct, but are merged, as 

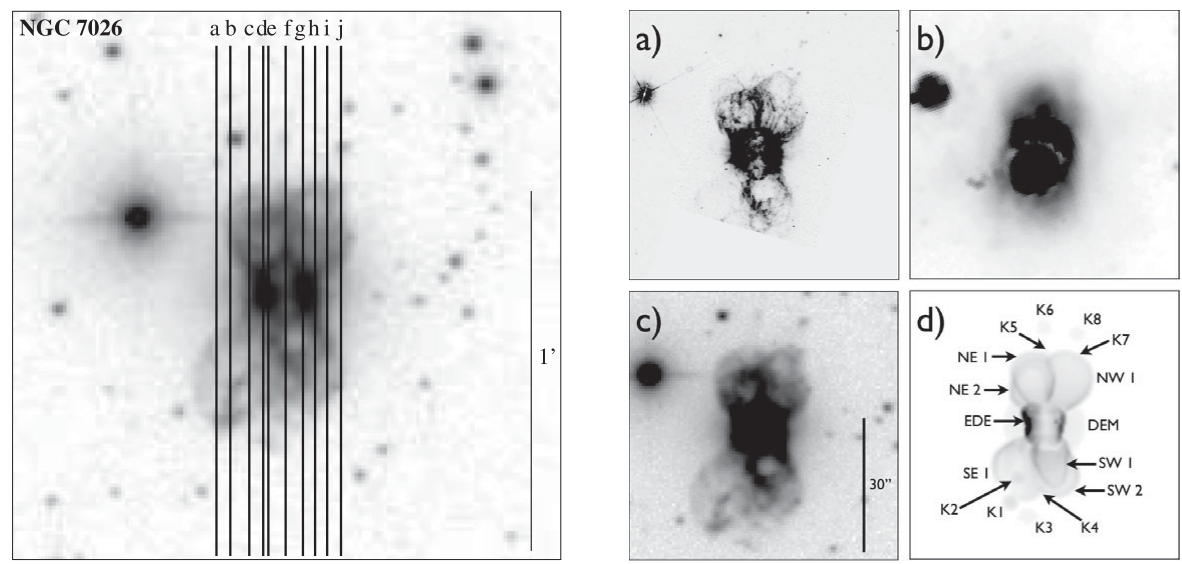

Figure 1. (left) $\mathrm{H} \alpha+[\mathrm{N} \mathrm{II}]$, SPM image with overlays of all slit positions acquired for NGC 7026. (right) Four different views of NGC 7026. a) HST [N II] image. b) $8 \mu \mathrm{m}$, Spitzer, archival image. c) MES-SPM, H $\alpha+[\mathrm{N} \mathrm{II}]$ image. d) Reconstruction of our Shape model with all major parts of the PN labeled, EDE: Equatorial Density Enhancement, DEM: Diffuse Equatorial Material, K1-K8: Knots 1-8. North is up, east is to the left and the scale is the same in all images.

represented in our Shape model. The complete lobe system is inclined 15 degrees with respect to the plane of the sky and has a position angle of 5 degrees. We measured an expansion velocity for the lobes of $V_{\exp }=150 \mathrm{~km} \mathrm{~s}^{-1}$. Using a distance of $2030 \mathrm{pc}$ to the nebula (Kwitter \& Henry 2006), we found an expansion age of $1.9 \times 10^{3}$ years.

The observed P-V diagrams for slit positions a and b show the $\mathrm{S}$ lobe is open (Figure 1, left). We represented this lack of emission in our Shape model by reducing the density to zero in the form of a wedge. The lack of X-ray emission in this region also indicates this $\mathrm{PN}$ has an open lobe to the SE.

NGC 7026 has a fast expanding equatorial density enhancement (EDE), with $V_{\exp }$ $=57 \mathrm{~km} \mathrm{~s}^{-1}$ as measured using slit $\mathrm{f}$. Outside of the EDE is fainter moving, diffuse equatorial material (DEM). In our Shape model we represented the EDE as a warped torus and the DEM as a torus filled with particles.

We found four sets of knots to the $\mathrm{N}$ and $\mathrm{S}$ of nebula, which appear to emanate from the lobes and we explored this possibility using Shape. We found that knots 1 and 8 do line up with a lobe, $\operatorname{knots} 4,5,6$, and 7 appear in near alignment with a lobe, but this is inconclusive, and knots 2 and 3 do not line up with a lobe at all. We found a range in deprojected velocities for knots $1-5$ and 7 of $V_{\text {dep }}=110-160 \mathrm{~km} \mathrm{~s}^{-1}$. Knot 6 had a measured $V_{\text {dep }}=81 \mathrm{~km} \mathrm{~s}^{-1}$ and knot 8 had a $V_{\text {dep }}=238.5 \mathrm{~km} \mathrm{~s}^{-1}$. We measured a mean expansion age for the knots of $\mathrm{t}=1.6 \times 10^{3} \mathrm{yr}$ with a standard deviation of $\sigma_{t}=$ $0.7 \times 10^{3} \mathrm{yr}$.

More details of this work will soon be published in Clark et al. (2012).

\section{References}

Cuesta, L., Phillips, J. P., \& Mampaso, A. 1996, A\&A, 313, 243

Gruendl, R. A., Guerrero, M. A., Chu, Y. H., \& Williams, R. M. 2006, ApJ, 653, 339

Kwitter, K. B. \& Henry, R. B. C. 2006, Gallery of Planetary Nebula Spectra, http:// web.williams.edu/Astronomy/research/PN/nebulae/index.php

Solf, J. \& Weinberger, R. 1984, A\&A, 130, 269

Steffen, W. \& López, J. A. 2006, Rev. Mexicana AyA, 42, 99

Steffen, W., Koning, N., Wenger, S., Morisset, C., \& Magnor, M., 2011, IEEE Transactions on Visualization and Computer Graphics, April 2011 (vol. 17 no. 4) pp. 454-465 